\title{
On the number of orbits of a group in two permutation actions
}

\author{
By
}

David M. EVANS and JoHanNes SIEMons

1. Introduction. Let $G$ be the symmetric group on the set $\Omega$ and suppose that $G$ also acts on some other set $\Delta$. When is it true that every subgroup of $G$ has at least as many orbits on $\Delta$ as on $\Omega$ ? This question is due to Alexander Zalesskii. We wish to thank him for bringing it to our attention.

In this note we give a complete - and more general - answer to his problem. First we need a definition, see also [2]: Let $(G, \Omega)$ be a permutation group, $F$ a field and $F \Omega$ the vector space of formal sums $\left\{\sum_{\omega \in \Omega} f_{\omega} \omega \mid f_{\omega}\right.$ in $F$, only finitely many $\left.f_{\omega} \neq 0\right\}$. Then $G$ acts naturally on $F \Omega$ and has $\left\{\sum_{\omega \in \Omega} f_{\omega} \omega \mid \sum_{\omega \in \Omega} f_{\omega}=0\right\}$, and $\left\langle\sum_{\omega \in \Omega} \omega\right\rangle$ if $\Omega$ is finite, as $G$-invariant subspaces. We say that $F \Omega$ is almost irreducible if there are no other $G$-invariant subspaces. Note that in this case $(G, \Omega)$ must be transitive.

Theorem A. Let $(G, \Omega)$ be a permutation group such that $F \Omega$ is almost irreducible for some field $F$. Suppose that $G$ acts also on some set $\Delta$. Then

(a) there is some $\delta$ in $\Delta$ such that $G_{\delta}$, the pointwise stabiliser of $\delta$, has a finite orbit $\Phi$ on $\Omega, \Phi \neq \Omega$ and the characteristic of $F$ does not divide $|\Phi|$, implies that

(b) for every subgroup $H$ of $G$ there are at least as many $H$-orbits on $\Delta$ as there are on $\Omega$.

If $G$ is finite and transitive on $\Delta$, then (b) implies (a).

In the case of finite groups almost irreducibility over the complex numbers is equivalent to double transitivity of the permutation action. For infinite groups the situation is a little more interesting. Results in [2] prove almost irreducibility of certain classes of infinite permutation modules. For example, if $(G, \Omega)$ is transitive and such that the pointwise stabiliser of every finite subset $X$ of $\Omega$ fixes only the points of $X$ then $F \Omega$ is almost irreducible, whatever the field $F$. Slightly more amusing is the case of the affine group AGL $(1, \mathbb{Q})$ acting on the rational line $\mathbb{Q}$. Here $F \mathbb{Q}$ is almost irreducible if and only if $F$ has non-zero characteristic. Presumably, in this case the conclusion of Theorem $A$ can be shown directly by looking at the possibilities for $\Delta$ and $H$.

The assumption of finiteness of the $G_{\delta}$-orbit is essential for Theorem A, this is shown by an example we give in Section 3. Returning to the original form of Zalesskii's question, however, we are able to prove 
Theorem B. Let $\Omega$ be a set, $G$ the symmetric group on $\Omega$ and suppose that $G$ acts also on some set $\Delta$. Then

(a) there is some $\delta$ in $\Delta$ such that $G_{\delta}$ is not transitive on $\Omega$, implies that

(b) for every subgroup $H$ of $G$, there are at least as many $H$-orbits on $\Delta$ as there are on $\Omega$.

If $G$ is transitive on $\Delta$, then (b) implies (a).

For the statement (b) we do not assume that the number of orbits is finite. Taking $H=1$, in particular, implies that $|\Omega| \leqq|\Delta|$. The proofs follow in Sections 2 and 4 below.

2. The proof of Theorem A. Let $G$ be a permutation group on a set $\Omega$ and let $F$ be a field. As above $F G$ and $F \Omega$ denote the vector spaces with bases $G$ and $\Omega$ respectively. There is also the usual multiplication defined on $F G$ which turns it into the group ring of $G$ over $F$. Then $F \Omega$ can be considered as an $F G$-module by extending the $G$-action on $\Omega$ in the obvious way.

One of the submodules mentioned above is the augmentation submodule $\operatorname{Aug}(F \Omega):=\left\{\sum_{\omega \in \Omega} f_{\omega} \omega \mid \sum_{\omega \in \Omega} f_{\omega}=0\right\}$. We define for any permutation group $G$ on $\Omega$ the subspace $\operatorname{Aug}_{G}(F \Omega)$ to be generated by the set $\{\omega-g \omega \mid \omega \in \Omega$ and $g \in G\}$. This clearly is an $F G$-submodule of $F \Omega$ with $\operatorname{Aug}(F \Omega) \geqq \operatorname{Aug}_{G}(F \Omega)$. If as usual $\operatorname{Aug}(F G)=\left\{\sum_{g \in G} f_{g} g \mid \sum_{g \in G} f_{g}=0\right\}$ is the augmentation ideal of $F G$, then $\operatorname{Aug}_{G}(F \Omega)=$ $\operatorname{Aug}(F G) \cdot F \Omega$.

Lemma. (i) If $M$ is an $F G$-submodule of $F \Omega$ then $F \Omega / M$ is trivial if and only if $M \geqq \operatorname{Aug}_{G}(F \Omega)$;

(ii) The dimension of $F \Omega / \operatorname{Aug}_{G}(F \Omega)$ is equal to the number of $G$-orbits on $\Omega$.

Proof. (i) If $g \in G$ and $\omega \in \Omega$, then $g(\omega+M)=\omega+M$ if and only if $\omega-g \omega \in M$.

(ii) Let $\Gamma$ be a system of representatives for the $G$-orbits on $\Omega$. Then $\left\{\gamma+\operatorname{Aug}_{G}(F \Omega) \mid \gamma \in \Gamma\right\}$ is an $F$-basis for $F \Omega / \operatorname{Aug}_{G}(F \Omega)$. For, if $\omega \in \Omega$ then there exist $g \in G$ and $\gamma \in \Gamma$ such that $g \gamma=\omega$. Thus $\gamma-\omega \in \operatorname{Aug}_{G}(F \Omega)$ and so $\omega+\operatorname{Aug}_{G}(F \Omega)$ $=\gamma+\operatorname{Aug}_{G}(F \Omega)$. Further, if $\sum_{\omega \in \Omega} a_{\omega} \omega$ belongs to $\operatorname{Aug}_{G}(F \Omega)$ then we have $\sum_{\omega \in A} a_{\omega}=0$ for each $G$-orbit $\Delta$. This implies linear independence. Hence $F \Omega / \operatorname{Aug}_{G}(F \Omega)$ has dimension $|\Gamma|$.

Proof of Theorem A. If $G_{\delta}$ is transitive on $\Omega$ and if $G$ is transitive on $\Delta$, then it is easy to see that $G_{\omega}$ is transitive on $\Delta$. Hence condition (b) is violated for $H=G_{\omega}$.

To prove that (a) implies (b) we may restrict $(G, \Delta)$ to the orbit containing $\delta$. Hence assume that $G$ is transitive on $\Delta$ and let $\Phi$ be the finite $G_{\delta}$-orbit on $\Omega, \Phi \neq \Omega$. For $\delta^{\prime} \in A$ we put $\Phi\left(\delta^{\prime}\right):=g \Phi$ where $g$ in $G$ is such that $g \delta=\delta^{\prime}$ (this does not depend on the choice of $g$ ). Define the linear map $\varphi: F \Delta \rightarrow F \Omega$ by setting $\varphi\left(\delta^{\prime}\right)=\sum_{\omega \in \Phi\left(\delta^{\prime}\right)} \omega$ for
$\delta^{\prime} \in A$. 
The hypotheses, about the characteristic of $F$, mean that the image of $\psi$ is not contained in $\operatorname{Aug}(F \Omega)$, and in the finite case, that it is not $\left\langle\sum_{\omega \in \Omega} \omega\right\rangle_{F}$. As $\varphi$ is an $F G$-homomorphism and since $F \Omega$ is almost irreducible, $\varphi$ must be surjective.

Now let $H$ be a subgroup of $G$. Then $\varphi\left(\operatorname{Aug}_{H}(F \Delta)\right)=\varphi(\operatorname{Aug}(F H) F \Delta)=$ $\operatorname{Aug}(F H) \varphi(F \Delta)=\operatorname{Aug}_{H}(F \Omega)$. Hence $\varphi$ induces a surjection from $F \Delta / \operatorname{Aug}_{H}(F A)$ onto $F \Omega / \operatorname{Aug}_{H}(F \Omega)$ and the claim follows from the lemma.

Variations: There are other ways to prove Theorem A which may be of independent interest:

1. Characters: We first proved Theorem $A$ for the finite case only and here one can argue as follows. Assume as above that $(G, \Omega)$ and $(G, \Delta)$ are transitive and let $\alpha$ and $\beta$ be the corresponding permutation characters. When $\chi_{0}=1, \chi_{1}, \chi_{2}, \ldots$ are the irreducible characters of $G$ then $\beta=1+n_{1} \chi_{1}+n_{2} \chi_{2}+\ldots$ and $\alpha=1+\chi_{1}$ as $G$ is doubly transitive on $\Omega$.

If $H$ is any subgroup of $G$ let $\pi=1+m_{1} \chi_{1}+m_{2} \chi_{2}+\ldots$ be the permutation character of $G$ acting on the cosets of $H$. Then, by reciprocity, the number of $H$-orbits on $\Omega$ and $\Delta$ are $\langle 1, \alpha\rangle_{H}=\langle\pi, \alpha\rangle_{G}$ and $\langle 1, \beta\rangle_{H}=\langle\pi, \beta\rangle_{G}$ respectively. Hence we are required to show that $1+m_{1} \leqq 1+n_{1} m_{1}+n_{2} m_{2}+\ldots$ which will certainly hold if $n_{1} \geqq 1$. But $n_{1}=0$ implies $1=\langle\alpha, \beta\rangle_{G}=\langle\alpha, 1\rangle_{G_{\delta}}$ so that $G_{\delta}$ is transitive on $\Omega$. Note that $1 \leqq n_{1}$ means that the character $\alpha$ is a constituent of $\beta$. This is a typical situation in finite orbit theorems, see also [3] and the survey in [4].

2. Another incidence map: Let $\overline{F \Omega}$ now be the space of functions $\Omega \rightarrow F$ whose image is finite. (Note: $F \Omega$ can be viewed as the space of functions whose support is finite.) A group $G$ on $\Omega$ acts on $\overline{F \Omega}$ via $(g f)(\omega)=f\left(g^{-1} \omega\right)$ and the dimension of the fixed point space is the number of $G$ orbits on $\Omega$. This approach to counting orbits has been suggested in [1].

The map $\varphi: F \Delta \rightarrow F \Omega$ defined above induces an $F G$-homomorphism $\varphi *: \overline{F \Omega} \rightarrow \overline{F \Delta}$ by $\left(\varphi^{*} f\right)(\delta)=f(\varphi(d))$. Clearly, if $\varphi$ is onto then $\varphi^{*}$ is injective and the result follows.

3. An example. We show that the hypothesis of finiteness of the $G_{\delta}$-orbit can not be removed from Theorem A. Our example is a highly transitive permutation group $(G, \Omega)$ and a permutation group $(G, \Delta)$ with the following properties: (i) $G_{\delta}$ has two orbits on $\Omega$, and (ii) there is an infinite, co-infinite subset $\Omega^{\prime}$ of $\Omega$ such that $G_{\left\{\Omega^{\prime}\right\}}$ is transitive on $\Delta$.

The universal, homogeneous, countable bipartite graph, equipped with a bipartition $(\Omega, \Delta)$ of its vertex set, is characterised by the following property:

(**) The bipartite graph $(\Omega, \Delta)$ is countably infinite and if $A, B$ are finite subsets of $\Omega$ (respectively $\Delta$ ) then there exists a vertex in $\Delta$ (respectively $\Omega$ ) which is adjacent to all vertices in $A$, and to none in $B$.

Let $(\Omega, A)$ be this graph and let $G$ be its automorphism group. Then $G$ acts faithfully and highly transitively on each of $\Omega$ and $\Delta$. Now, there exists an infinite, co-infinite subset $\Omega^{\prime}$ of $\Omega$ such that each of $\left(\Omega^{\prime}, \Delta\right)$ and $\left(\Omega \backslash \Omega^{\prime}, \Delta\right)$ has property (**). A simple back-and-forth argument then establishes that $G_{\left(\Omega^{\prime}\right)}$ is transitive on $\Delta$. (One way of seeing the existence 
of such a subset $\Omega^{\prime}$ is to recall that the graph can be constructed "randomly": Enumerate all pairs $\Omega \times \Delta$ and toss a fair coin to decide (independently) which are edges in the graph. With probability 1 , the resulting graph has property $(* *)$. So now do this construction again, but start off with $\Omega$ partitioned into two infinite parts $\Omega^{\prime}$ and $\Omega^{\prime \prime}$. Again with probability 1 , each of $\left(\Omega^{\prime}, \Delta\right),\left(\Omega^{\prime \prime}, \Delta\right),(\Omega, \Delta)$ has property (**).)

4. Proof of Theorem B. Let $G$ be the symmetric group on $\Omega$ acting also on some sets $\Delta$. If $G$ is transitive on $\Delta$ and if $G_{\delta}$ is transitive on $\Omega$ it is clear that $G_{\omega}$ is transitive on $\Delta$. Hence $H=G_{\omega}$ violates condition (b).

We now come to the converse. As before it suffices to assume that $G$ is transitive on $\Delta$. Let $\Sigma$ be a $G$-orbit on $\Omega \times \Delta$ and for $\delta \in \Delta$ let $\Sigma(\delta)=\{\omega \in \Omega \mid(\omega, \delta) \in \Sigma\}$. Note that by assumption $\Sigma(\delta) \neq \Omega$ and also $\Sigma(g \delta)=g \Sigma(\delta)$ for $g \in G$. Define the $G$-relation $\approx$ on $\Delta$ by $\delta \approx \delta^{\prime}$ if and only if $\Sigma(\delta)=\Sigma\left(\delta^{\prime}\right)$. Let $\Delta^{*}$ be the quotient $G$-set $\Delta / \approx$. Clearly, if $H \leqq G$ then the number of orbits of $H$ on $\Delta$ is not less than the number of orbits of $H$ on $A^{*}$ (if $\delta / \approx$ and $\delta^{\prime} / \approx$ lie in different $H$-orbits then there does not exist $h \in H$ with $h \Sigma(\delta)=h \Sigma\left(\delta^{\prime}\right)$, so in particular $\delta$ and $\delta^{\prime}$ lie in different $H$-orbits).

Thus it will suffice to show that if $H \leqq G$ then the number of $H$-orbits on $A^{*}$ is not less than the number of $H$-orbits on $\Omega$. Note that $\left(G, \Delta^{*}\right)$ is permutation equivalent to $G$ acting on the set $\Omega^{\lambda, \mu}=\{X \subseteq \Omega|| X|=\lambda,| \Omega \backslash X \mid=\mu\}$, where $\lambda=|\Sigma(\delta)|$ and $\mu=|\Omega \backslash \Sigma(\delta)|$. Note that $\lambda, \mu \neq 0$ and that we can assume $\lambda \leqq \mu$. Let $H \leqq G$ and let $\left(O_{i} \mid i \leqq v\right)$ be the $H$-orbits on $\Omega$ (here $v$ is some initial ordinal), and arranged so that $\left|O_{\alpha}\right| \leqq\left|O_{\beta}\right|$ if $\alpha \leqq \beta$.

Case $1 . \lambda<|\Omega|$. Let $\alpha \leqq v$ be minimal such that $\left|\cup\left\{O_{i} \mid i \leqq \alpha\right\}\right| \geqq \lambda$. Let $Z_{\alpha}$ be a set of size $\lambda$ with $\cup\left\{O_{i} \mid i<\alpha\right\} \subseteq Z_{\alpha} \leqq \cup\left\{O_{i} \mid i \leqq \alpha\right\}$, and let $a_{i} \in O_{i}$ (and $a_{\alpha} \in Z_{\alpha}$ ). If $i \neq \alpha$ define sets $Z_{i}$ as follows:

$$
\begin{aligned}
& \text { if } i>\alpha \text { then } Z_{i}=\left(Z \backslash\left\{a_{\alpha}\right\}\right) \cup\left\{a_{i}\right\} ; \\
& \text { if } i<\alpha \text { then } Z_{i}=\left(Z \backslash\left\{a_{i}\right\}\right) \cup\{b\}, \text { where } b \in \Omega \backslash Z_{\alpha} .
\end{aligned}
$$

Then $\left(Z_{i} \mid i \leqq v\right)$ lie in different $H$-orbits.

In the remaining two cases $\lambda$ is of course infinite.

Case 2. $\mu=\lambda=|\Omega|$, and $\left|O_{v}\right|=|\Omega|$. The above construction works automatically if $\alpha<v$. To make it work if $\alpha=v$ we need only to ensure that $\left|O_{v} \backslash Z_{v}\right|=\mu$, which is not a problem.

Case 3. $\mu=\lambda=|\Omega|$, and $\left|O_{v}\right| \neq|\Omega|$. In this case, $v=|\Omega|$, and so there are $2^{|\Omega|}$ $H$-orbits on $\Omega^{\lambda, \lambda}$. This completes the proof of Theorem $\mathbf{B}$.

\section{References}

[1] P. Cameron, An orbit theorem for Steiner triple systems. Proceedings of 13th British Combinatorial Conference, to appear.

[2] A. R. Camina and D. M. Evans, Some infinite permutation modules. Quart. J. Math. Oxford Ser. (2) 42, 15-26 (1991). 
[3] A. R. CAMINA and J. SIEMONS, Intertwining automorphisms in finite incidence structures. Linear Algebra Appl. 117, 25-34 (1989).

[4] A. WAGNER, Orbits on finite incidence structures. Sympos. Math. Istit. Naz. Alta Mat. 28, 219-229 (1984).

Eingegangen am 14. 2. 1992

Anschrift der Autoren:

David M. Evans

Johannes Siemons

School of Mathematics

University of East Anglia

Norwich NR4 7TJ

England 